\title{
CÂNCER DE MAMA E DISFUNÇÃO ENDOTELIAL
}

\author{
Carolina Oliveira Nastri*, Wellington de Paula Martins, Francisco José Candido dos Reis, Rui Alberto Ferriani \\ Trabalho realizado pelo Departamento de Ginecologia e Obstetrícia do Hospital das Clínicas da Faculdade de Medicina de Ribeirão Preto da \\ Universidade de São Paulo, Ribeirão Preto, SP
}

\author{
*Correspondência: \\ Rua Manoel Ache, 920 apto \\ 1904 \\ Jardim Irajá - Ribeirão \\ Preto - SP \\ CEP 14020-590 \\ Tel: (16) 81174090 \\ canastri@hotmail.com
}

\begin{abstract}
RESUMO
Entre as mulheres brasileiras a principal causa de mortalidade são as doenças cardiovasculares, seguida em freqüência pelo câncer, sendo o de mama o mais comum. É bastante conhecida a associação de câncer com eventos tromboembólicos, mas pouco estabelecida sua relação com os demais eventos cardiovasculares. Para estudar estes eventos desde suas alterações primordiais, como a lesão e disfunção endotelial e a formação da placa aterosclerótica, vários métodos têm sido utilizados. Dentre eles, a dosagem sérica de $\mathrm{P}$ e E-selectina e do fator de von Willebrand são relevantes devido à associação tanto com o risco cardiovascular quanto com o processo de progressão e formação de metástase do câncer de mama. Outro método de avaliação da função endotelial é a medida da dilatação da artéria braquial mediada por fluxo, que cada vez mais ganha popularidade devido à sua natureza não-invasiva e a comprovação de sua associação com a disfunção endotelial e risco de eventos cardiovasculares. Buscamos, através desta revisão, condensar o que houve de mais relevante nestes últimos anos sobre a associação de câncer, em especial o de mama, com lesão endotelial e risco cardiovascular.
\end{abstract}

UnITERMOS: Neoplasias mamárias. Doenças cardiovasculares. Endotélio.

\section{INTRODUÇÃO}

As doenças do sistema circulatório correspondem à principal causa de morte no Brasil, tendo ocorrido 285.543 mortes por essa causa (total de 1.024.073) durante 0 ano de 2004, segundo dados mais recentes do Ministério da Saúde!. Em segundo lugar aparecem as mortes por câncer, sendo o de mama o mais incidente entre as mulheres, excluindo os tumores de pele não melanoma. $O$ câncer de mama apresenta incidência crescente mundialmente sendo, no Brasil, em 2006, o risco estimado pelo INCA ${ }^{2}$ em 52 casos novos por 100.000 mulheres.

A associação de câncer e trombofilia foi descrita pela primeira vez em 1.865, pelo clínico francês Armand Trousseau³, porém somente nas três últimas décadas maior atenção foi dada à questão da associação molecular entre a ativação da coagulação e a progressão do câncer ${ }^{4}$.

\section{Doença cardiovascular e aterosclerose}

A maneira mais efetiva de prevenir a trombose arterial e, consequentemente, eventos cardiovasculares, é a prevenção da aterosclerose ${ }^{5,6}$. Em 1912, dois patologistas americanos da Universidade de Pittsburgh publicaram um resumo de seus estudos sobre artérias humanas e concluíram: "É bastante inútil discutir questões a respeito do desenvolvimento da esclerose da íntima se somente discutirmos sobre os estágios avançados da doença. Se pretendermos avançar na complexa questão da aterosclerose, deve-se preocupar em entender a lesão desde seus primórdios"7. Tais autores julgavam haver forte indicação de que a produção de tecido pela íntima dos vasos se devia ao resultado da irritação direta deste tecido pela presença de infecção ou toxinas, ou ainda pela estimulação de produtos da degeneração primária desta camada.
A aterosclerose é um processo que acomete artérias de médio e grande calibre, e se inicia com o aumento da expressão de moléculas de adesão pelas células endoteliais como resposta ao fluxo turbulento em um ambiente sérico desfavorável do ponto de vista do perfil lipídico. Não é mais considerada simplesmente uma desordem secundária a anormalidades no metabolismo lipídico, atualmente é bem descrito o papel da inflamação desde os estágios iniciais da formação da placa aterosclerótica: na promoção da mitogênese, proliferação da matriz intracelular, angiogênese e desenvolvimento das células espumosas ${ }^{8}$. A proteína $\mathrm{C}$ reativa $(\mathrm{pCr}$ ) e o fibrinogênio são marcadores associados a patogenia da aterosclerose em modelos animais e seus efeitos incluem diminuição do óxido nítrico (NO) e prostaciclinas endoteliais?. A evoluçãa da placa aterosclerótica é marcada pelo crescimento gradual, conseqüente à deposição de células espumosas, com a placa fibrosa definitiva sendo elaborada lentamente pela proliferação de células musculares lisas e de matriz intracelular. Entretanto, o infarto miocárdico muitas vezes ocorre em artérias com pouca oclusão prévia, sugerindo um crescimento abrupto destas placas. A oclusão trombótica aguda se dá como consequeência da súbita ruptura da placa aterosclerótica através de fissura espontânea, quando exposta ao estresse do turbilhonamento em locais de estenose ou de ramificação arterial. Tal dano provoca a denudação endotelial estimulando a formação de trombo local ${ }^{10}$, este pode obstruir parcial ou totalmente a parede do vaso, sendo responsável pelo evento clínico.

A lesão da parede do vaso caracteriza-se por perda de continuidade do endotélio e pode ser causada em algumas situações: angioplastia, estresse hemodinâmico, tabagismo, hipercolesterolemia ou por enzimas liberadas por plaquetas e leucócitos 6 . A perda de células 
endoteliais expõe o subendotélio às plaquetas e aos fatores da coagulação. A adesão plaquetária é mediada por diversas substâncias, entre elas o fvW. O endotélio exposto a endotoxinas, citocinas, trombina e hipóxia acaba promovendo a coagulação e regulando o fluxo sangüíneo através de substâncias vasoconstrictoras, como endotelinas e vasodilatadoras, prostaciclinas e $\mathrm{NO}^{6}$.

\section{Função endotelial}

A disfunção endotelial é observada em estágios iniciais na maioria das doenças cardiovasculares e surge como um fator preditor independente para eventos clínicos na doença coronariana, hipertensão e falência cardíaca. Neste contexto, a avaliação da função endotelial passa de um intermediário na avaliação do benefício de tratamentos cardiovasculares e emerge como um alvo farmacológico direto".

\section{Marcadores de função endotelial}

\section{Fator de von Willebrand}

O fator de von Willebrand (fvW) é uma glicoproteína sintetizada pelo endotélio e é responsável pela mediação entre a adesão plaquetária ao subendotélio durante o processo de hemostasia primária. O aumento dos níveis séricos de fvW é associado com maior risco de eventos clínicos da doença cardiovascular, principalmente tromboembolismo, infarto agudo do miocárdio e acidentes vasculares cerebrais ${ }^{12}$. Níveis elevados de fvW foram também descritos em alguns tipos de cânceres (cabeça e pescoço, próstata) e bem correlacionados ao câncer de mama ${ }^{13}$. Em um estudo foi comparada a dosagem de fvW em 128 pacientes com câncer de mama, em 47 pacientes com doença mamária benigna e em 27 controles. A média e desvio padrão da dosagem de fuW no grupo controle foi de 130,6 $\pm 45 \mathrm{U} / \mathrm{dl}$, nas pacientes com doença benigna $148,4 \pm 59 \mathrm{U} / \mathrm{dl}$ e nas com câncer |70,7 $\pm 78 \mathrm{U} / \mathrm{dl}$, sendo significativa a diferença encontrada entre as pacientes com câncer e os outros dois grupos ${ }^{13}$. Durante a fase ativa da angiogênese tumoral, são sintetizados fator de crescimento do endotélio vascular (VEGF) e fator de crescimento de fibroblasto (FGF-2), que contribuem para a proliferação e diferenciação do endotélio vascular, o maior produtor de fvW, resultando em aumento dos níveis séricos de fvW' ${ }^{14}$. O fvW também contribui para a adesão entre plaquetas e células tumorais, através das integrinas, sendo este um passo importante na determinação de como a coagulação sanguínea e a agregação plaquetária podem ser mediadas pela célula tumoral. Outro resultado importante da ligação entre plaquetas e células neoplásicas é a capacidade que esta adquire de migrar, auxiliando no processo de metástase. De fato, são demonstradaa taxas de fvW sérico mais altas em pacientes com câncer de estadios mais avançados do que em estadios iniciais ${ }^{13}$.

O aumento dos níveis séricos do fvW tem sido descrito como fator de risco para desfechos clínicos da doença cardiovascular como tromboembolismo, infarto agudo do miocárdio e acidentes vasculares cerebrais $^{12}$. Em uma metanálise foi confirmada a relação do frW com doença coronariana, sendo verificado que o grupo constituído das pessoas com níveis séricos acima do percentil 67 apresentou $50 \%$ mais risco de doença cardiovascular quando comparado ao grupo das pessoas com níveis séricos abaixo do percentil $33^{15}$.

\section{Selectinas}

A família das selectinas é composta por três membros: L-selectina, P-selectina e E-selectina, sendo esta última a única encontrada apenas no endotélio. Esta especificidade proporciona uma oportunidade para o estudo de aspectos fisiopatológicos do endotélio na doença cardiovascular e inflamatória"!.

Tanto a E-selectina como a P-selectina são moléculas expressas na superfície do endotélio quando estimulado por algumas citocinas inflamatórias (interleucinas I e I0, fator de necrose tumoral- $\alpha$ ); a $P$ selectina pode ser também encontrada na superfície de plaquetas ativadas. Quando expressas, as selectinas promovem a adesão do leucócito ao endotélio. A fração solúvel da E-selectina é proveniente da clivagem proteolítica da molécula expressa na membrana celular"'. O papel destas moléculas na história natural do câncer não é completamente esclarecido, mas acredita-se que desempenhe função fundamental no processo de disseminação por via hematogênica do tumor, promovendo a adesão da célula neoplásica ao endotélio ${ }^{16}$.

Níveis séricos aumentados de E-selectina são associados com estágios avançados de câncer de mama e com a presença de células tumorais circulantes ${ }^{17}$. Em outra publicação, foi obtida média da dosagem sérica de E-selectina significantemente maior em pacientes com câncer de mama quando comparado com mulheres saudáveis: 73,7 e $36,3 \mathrm{ng} / \mathrm{ml}$, respectivamente, além de significativa correlação positiva com o estadiamento clínico ${ }^{16}$. Além disso, o aumento da concentração sérica de E-selectina foi associado com tabagismo, hipertensão, hipercolesterolemia, doença arterial coronariana, doença cérebrovascular e Diabetes mellitus ${ }^{18}$. Acredita-se que o aumento da concentração sérica observada na hipertensão arterial deva refletir a disfunção endotelial, mas não necessariamente, predizer o desenvolvimento de lesão de órgão alvo ${ }^{19}$, pois, diferentemente do fvW, a E-selectina está mais relacionada com a regeneração do endotélio após o dano tissular provocado pela progressão da doença ${ }^{5}$. Apesar da capacidade da dosagem de E-selectina em predizer eventos adversos e suas implicações clínicas serem bastante controversas, pode-se dizer que, em pacientes sofrendo de um evento cardiovascular agudo, a E-selectina freqüentemente se encontra aumentada, quando comparado com controles pareados por sexo e idade ${ }^{18}$.

Outra selectina, a P-selectina, também está presente em maior concentração sérica em pacientes com câncer de mama ${ }^{20}$. Quanto ao seu papel na disseminação neoplásica, sabe-se que esta molécula, expressa pelas células endoteliais estimuladas, promove a adesão de células tumorais circulantes mediando a disseminação tumoral por via hematogênica, enquanto a interação dessas células com a P-selectina expressa em plaquetas ativadas, supre o tumor com fatores de crescimento e substâncias mitogênicas ${ }^{21}$. Além do câncer, esta molécula é encontrada em níveis elevados em situações como diabetes, hipertensão, tabagismo e hipercolesterolemia e parece ser capaz de predizer a ocorrência de um evento cardiovascular adverso ${ }^{22}$.

\section{Dilatação mediada por fluxo dependente do endotélio (DMF)}

O estudo da doença vascular pré-clínica se tornou mais viável através da aplicação de técnicas ultra-sonográficas não-invasivas. A avaliação da dilatação da artéria braquial foi originalmente descrita em 1992 e é realizada por ultra-sonografia após oclusão arterial temporária 
através de torniquete aplicado ao membro superior ${ }^{23,24}$. Retirado o torniquete, é então observada a dilatação do vaso em resposta a tensão de cisalhamento associada ao súbito aumento de fluxo, resposta esta dependente de $\mathrm{NO}$ e independente de prostaciclinas ${ }^{24}$. O NO é produzido no endotélio via L-arginina por enzimas óxido nítrico sintases, a ativação desta via se dá através de estímulos como hipóxia, acetilcolina, bradicinina e serotonina. O NO promove vasodilatação direta, inibe a liberaçãa de fatores vasoconstritores, proliferação de células musculares lisas, adesão leucocitária e agregação plaquetária 25 .

Uma porcentagem de dilatação menor que o normal é observada tanto em pacientes com doença aterosclerótica como naqueles em risco para tal: mulheres pós-menopausadas, hipertensos, diabéticos, obesos, sedentários, tabagistas ativos e passivos e naqueles com aumento dos níveis séricos de colesterol e homocisteína. O controle destes fatores de risco melhora a função endotelial ao aumentar a DMF, o que expressa um aumento na liberação de $\mathrm{NO}^{26}$. Em um estudo que seguiu por cinco anos 74 pacientes com dor torácica mostrou que aqueles com DMF normal, considerada como dilatação maior ou igual a $10 \%$, apresentaram menor número de eventos coronarianos e foram submetidos a angioplastia e/ou revascularização cardíaca menos frequentemente. A análise destes dados mostrou sensibilidade de 86\%, especificidade de $51 \%$ e valor preditivo negativo de $85 \%$, considerando como positivo o exame que resultou em menos de $10 \%$ de dilatação na artéria braquial e o desfecho positivo como infarto agudo do miocárdio, angioplastia ou revascularização do miocárdio ${ }^{27}$.

Esse padrão de achados é semelhante aos identificados para fvW e, além disso, ambos são capazes de predizer o desenvolvimento de eventos cardiovasculares maiores. É demonstrada, inclusive, correlação inversa entre os dois, ou seja, aqueles indivíduos com os maiores níveis séricos de fvW apresentam também DMF alterada, enquanto que aqueles com baixos níveis de fvW apresentam DMF normal ${ }^{5}$.

\section{Células endoteliais circulantes}

O achado de células endoteliais livres na circulação sangüínea é raro em indivíduos saudáveis, e tem sido indicada como um estágio final do injúrio endotelial. De fato, sua presença é fortemente correlacionada com doença coronariana aguda; o aumento de seus níveis nas primeiras 48 horas após um evento coronariano é também considerado fator preditivo de morte. Este promissor marcador de dano endotelial apresenta boa correlação com outros marcadores de função endotelial, como fvW e DMF, em pacientes com síndrome coronariana aguda. Tais achados sugerem que o dano endotelial agudo, como o manifestado pelo aumento das CEC, pode ser o principal determinante da alteração da vasodilatação mediada por fluxo, provavelmente devido à diminuição da biodisponibilaidade de NO. Na doença coronariana estável, há um menor grau de injúria endotelial, representada pela menor quantidade de CEC, entretanto, a mantida disfunção endotelial e inflamação, indicadas pelos altos níveis de fvW e IL-6 em relação aos controles, pode ser refletida pela alteração da FMD nesses pacientes ${ }^{28}$.

\section{Considerações finais}

Pacientes com câncer que desenvolvem tombose venosa apresentam expectativa de vida reduzida: indivíduos que apresentam um evento trombótico têm risco de morte relacionada ao evento quatro a oito vezes maior se possuírem também diagnóstico de câncer. Estes indivíduos apresentam sobrevida reduzida quando comparados aos pacientes oncológicos cuja doença não curse com tais episódios. Este aumento na mortalidade reflete, provavelmente, não apenas mortes relacionadas ao tromboembolismo, mas também um curso mais agressivo destes tumores ${ }^{29}$.

São atualmente conhecidos diversos marcadores séricos de risco cardiovascular que se apresentam comprovadamente aumentados em mulheres portadoras de câncer de mama, como E e P-selectina e fator de von Willebrand. Na literatura há extensa correlação entre neoplasias malignas e trombose venosa, porém pouca informação sobre os efeitos dos produtos tumorais sobre o endotélio e sua influência quanto ao risco cardiovascular.

\section{Conflito de interesse: não há}

\section{SUMMARY}

\section{BREAST CANCER AND ENDOTHELIAL DYSFUNCTION}

The main cause of death among Brazilian women is cardiovascular disease followed by cancer with breast cancer as the most incident. The relationship between cancer and thrombosis is well known, although its association with other cardiovascular events is poorly understood. In order to study these events from the earliest findings such as endothelial injury and dysfunction and the evolving atherosclerotic plaque, many methods are currently being used. Among these methods, E-and P-selectin and the von Willebrandfactor have been associated, either with cardiovascular risk or with breast cancer growth and metastasis. Brachial artery flowmediated dilatation is a tool available that emerged in the last decade due to its noninvasive nature and its clear association with endothelial dysfunction and cardiovascular risk. The aim of this revision is to bring the newest and most relevant updates about the association of breast cancer, endothelial injury and cardiovascular risk. [Rev Assoc Med Bras 2008; 54(5): 467-70]

KEY wORDS: Breast neoplasms. Cardiovascular diseases. Endothelium.

\section{REFERÊNCIAS}

I. Ministério_da_Saúde. Freqüência de óbitos por doenças do aparelho circulatório - [citado 2008 jun 3]. Disponível em: http:// portal.saude.gov.br/portal/svs/visualizar_texto.cfm?idtxt=2442I .

2. INCA (Instituto Nacional do Câncer). Estimativa 2006: incidência de câncer no Brasil [citado 2008 jun 3]. Disponível em: http://www.inca.gov.br/estimativa/2006/index.asp?link = conteudo view.asp\&ID $=5$.

3. Trousseau A. Clinique Médicale de l'Hotel-Dieu de Paris. Paris: JB Ballière et Fils; 1865.

4. Rickles FR. Mechanisms of cancer-induced thrombosis in cancer. Pathophysiol Haemost Thromb. 2006;35: 103- 10.

5. Blann AD. Assessment of endothelial dysfunction: focus on atherothrombotic disease. Pathophysiol Haemost Thromb. 2003;33:256-61

6. Crowther MA. Pathogenesis of atherosclerosis. Hematology Am Soc Hematol Educ Program. 2005:436-4I.

7. Klotz $\mathrm{O}$, Manning M. Fatty streaks in intima of arteries. J Pathol Bacteriol. 1912;16:211-20.

8. Libby P, Theroux P. Pathophysiology of coronary artery disease. Circulation. 2005; 1 1 1:3481-8. 
9. Danenberg HD, Szalai AJ, Swaminathan RV, Peng L, Chen Z, Seifert P, et al. Increased thrombosis after arterial injury in human C-reactive proteintransgenic mice. Circulation. 2003; 108:5 12-5.

10. Davies MJ. A macro and micro view of coronary vascular insult in ischemic heart disease. Circulation. 1990;82:1138-46.

I I. Joannides R, Bellien J, Thuillez C. Clinical methods for the evaluation of endothelial function: a focus on resistance arteries. Fundam Clin Pharmacol. 2006;20:311-20.

12. Blann AD. Plasma von Willebrand factor, thrombosis, and the endothelium: the first 30 years. Thromb Haemost. 2006;95:49-55.

13. Rohsig LM, Damin DC, Stefani SD, Castro CG Jr, Roisenberg I, Schwartsmann G. von Willebrand factor antigen levels in plasma of patients with malignant breast disease. Braz J Med Biol Res. 2001;34:1 125-9.

14. Gil-Bazo I, Catalan V, Paramo J, Quero C, Escriva de Romani S, PerezOchoa $A$, et al. Von Willebrand factor as an intermediate between hemostasis and angiogenesis of tumor origin. Rev Med Univ Navarra. 2003;47:22-8.

15. Whincup PH, Danesh J, Walker M, Lennon L, Thomson A, Appleby P, et al. von Willebrand factor and coronary heart disease: prospective study and meta-analysis. Eur Heart J. 2002;23: I 764-70.

16. Sheen-Chen SM, Eng HL, Huang CC, Chen WJ. Serum levels of soluble E-selectin in women with breast cancer. Br J Surg. 2004;91: I 578-8 I.

17. Silva HC, Garcao F, Coutinho EC, CF DEO, Regateiro FJ. Soluble VCAMI and E-selectin in breast cancer: relationship with staging and with the detection of circulating cancer cells. Neoplasma. 2006;53:538-43.

18. Roldan V, Marin F, Lip GY, Blann AD. Soluble E-selectin in cardiovascular disease and its risk factors. A review of the literature. Thromb Haemost. 2003;90: 1007-20

19. Galen FX. Cell adhesion molecules in hypertension: endothelial markers of vascular injury and predictors of target organ damage? J Hypertens. 2002;20:813-6.

20. Blann AD, Gurney D, Wadley M, Bareford D, Stonelake P, Lip GY. Increased soluble P-selectin in patients with haematological and breast cancer: a comparison with fibrinogen, plasminogen activator inhibitor and von Willebrand factor. Blood Coagul Fibrinolysis. 200 I; 12:43-50.
21. Chen M, Geng JG. P-selectin mediates adhesion of leukocytes, platelets, and cancer cells in inflammation, thrombosis, and cancer growth and metastasis. Arch Immunol Ther Exp (Warsz). 2006;54:75-84.

22. Blann AD, Nadar SK, Lip GY. The adhesion molecule P-selectin and cardiovascular disease. Eur Heart J. 2003;24:2166-79.

23. Celermajer DS, Sorensen KE, Gooch VM, Spiegelhalter DJ, Miller OI, Sullivan ID, et al. Non-invasive detection of endothelial dysfunction in children and adults at risk of atherosclerosis. Lancet. 1992;340: I | | I-5.

24. Corretti MC, Anderson TJ, Benjamin EJ, Celermajer D, Charbonneau F, Creager MA, et al. Guidelines for the ultrasound assessment of endothelial-dependent flow-mediated vasodilation of the brachial artery: a report of the International Brachial Artery Reactivity Task Force. J Am Coll Cardiol. 2002;39:257-65.

25. Joannides R, Haefeli WE, Linder L, Richard V, Bakkali EH, Thuillez C, et al. Nitric oxide is responsible for flow-dependent dilatation of human peripheral conduit arteries in vivo. Circulation. 1995;91:13|4-9.

26. Esper RJ, Nordaby RA, Vilarino JO, Paragano A, Cacharron JL, Machado RA. Endothelial dysfunction: a comprehensive appraisal. Cardiovasc Diabetol. 2006;5:4.

27. Neunteufl T, Heher S, Katzenschlager R, Wolfl G, Kostner K, Maurer G, et al. Late prognostic value of flow-mediated dilation in the brachial artery of patients with chest pain. Am J Cardiol. 2000;86:207- I0.

28. Lee KW, Blann AD, Lip GY. Inter-relationships of indices of endothelial damage/dysfunction [circulating endothelial cells, von Willebrand factor and flow-mediated dilatation] to tissue factor and interleukin-6 in acute coronary syndromes. Int J Cardiol. 2006; I I 1:302-8.

29. Lee AY, Levine MN. Venous thromboembolism and cancer: risks and outcomes. Circulation. 2003; 107:117-21.

Artigo recebido: 02/10/07

Aceito para publicação: 19/03/08 